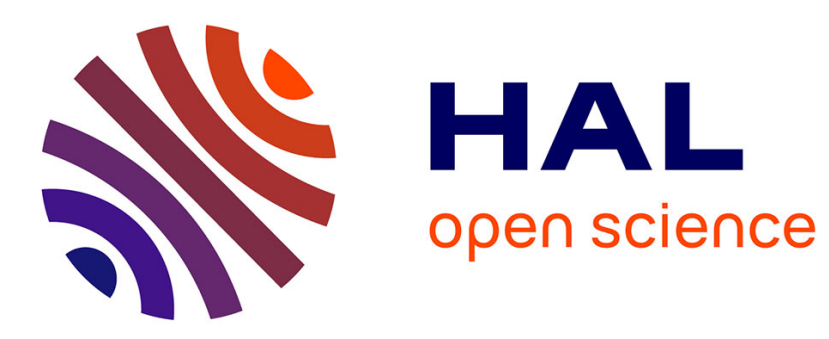

\title{
Nonlinear filtering for ultra-tight gnss/ins integration
} Carles Fernandez Prades, Pau Closas, Jordi Vilà Valls

\section{To cite this version:}

Carles Fernandez Prades, Pau Closas, Jordi Vilà Valls. Nonlinear filtering for ultra-tight gnss/ins integration. ICC 2010 - IEEE International Conference on Communications, May 2010, Le Cap, South Africa. pp.n.c. hal-00448865

\section{HAL Id: hal-00448865 https://hal.science/hal-00448865}

Submitted on 20 Jan 2010

HAL is a multi-disciplinary open access archive for the deposit and dissemination of scientific research documents, whether they are published or not. The documents may come from teaching and research institutions in France or abroad, or from public or private research centers.
L'archive ouverte pluridisciplinaire HAL, est destinée au dépôt et à la diffusion de documents scientifiques de niveau recherche, publiés ou non, émanant des établissements d'enseignement et de recherche français ou étrangers, des laboratoires publics ou privés. 


\title{
NONLINEAR FILTERING FOR ULTRA-TIGHT GNSS/INS INTEGRATION
}

\author{
Carles Fernández-Prades*, Pau Closas*, Jordi Vilà-Valls** \\ * Centre Tecnològic de Telecomunicacions de Catalunya (CTTC) \\ Av. Carl Friedrich Gauss 7, 08860 Castelldefels, Spain. E-mail:\{carles.fernandez, pau.closas\}@cttc.cat \\ ** GIPSA-Lab, Grenoble Institute of Technology (INPG) \\ BP 46 - 38402 Saint Martin d'Hères, France. E-mail: jordi.vilavalls@gipsa-lab.grenoble-inp.fr
}

\begin{abstract}
This paper considers the problem of ultra-tight GNSS/INS integration. We propose a new approach, deriving the direct relation between Inertial Measurement Unit (IMU) measurements and synchronization parameters, used in the trilateration algorithm to compute the position of the receiver. We take into account the IMU's eventual biased behavior by introducing it into the state representation. We use a recently-developed, square-root derivative-free Gaussian nonlinear filter to solve the estimation problem.
\end{abstract}

Index Terms - GNSS/INS integration, cubature rules, synchronization, square-root Cubature Kalman filter

\section{INTRODUCTION}

Accuracy and availability are the main objectives of a navigation system. Since Global Navigation Satellite Systems (GNSS) performance is not sufficient for all applications in geodesy and navigation, it is common to hybridize them with Inertial Navigation Systems (INS), in order to combine the only short-time stability of an inertial sensor and the longtime stability but noise behavior of a GNSS receiver, taking advantage of their complementary nature.

In most GNSS/INS integrations the position and velocity estimates of a GNSS receiver are used as observations in a navigation filter for the estimation of INS errors, making possible a reduction of GNSS-noise, bridging of GNSS outages, and even GNSS measurements are also usable when fewer than four satellites are tracked. This combination, known as loosely coupled integration, provides good performance combined with simplicity of integration. The mathematical problem posed by this integration can be cast into a nonlinear filter, that traditionally has been solved by means of the Extended Kalman Filter (EKF).

In case of high dynamic applications or in jamming environments, information about the receiver dynamics measured by the inertial sensor can be used to enhance the

This work has been partially supported by the Spanish Science and Technology Commission: CENIT2007-2002 (TIMI) and TEC2008-02685/TEC (NARRA), and by the European Commission in the framework of the COST Action IC0803 (RFCSET) and the FP7 Network of Excellence in Wireless COMmunications NEWCOM++ (contract n. 216715).
pseudorange/Doppler estimations of the GNSS receiver. This principle is known as tightly coupled integration, and it poses a nonlinear problem. Examples of this approach can be found in [1]-[3], making use of the UKF, Sigma-Point Kalman Filter (SPKF), and Particle Filtering (PF) methods. In [4], the approach is cast into a convex, linearly constrained leastsquares problem and solved via dual decomposition.

An extension of this concept is the ultra-tightly coupled integration. In this case the integrating navigation filter is implemented as one element of the receiver tracking loop. An optimal controlling value for the numerical oscillator can be computed by using inertial information in combination with the in-phase (I) and quadrature (Q) signals at the receiver. Examples of this approach can be found in [5][7], where the errors in position and velocity between the measured and estimated values act as a link between I \& Q samples and INS measurements, and lead to slight modifications of the tracking loops.

This paper proposes a new approach to ultra-tight GNSS/INS integration by deriving the evolution of the synchronization parameters from the INS measurements, considering the eventual biased behavior of the latter, and taking advantage of the numerical efficiency of the Squareroot Cubature Kalman Filter (SCKF), a nonlinear Gaussian filter recently developed [8], that could substitute the structure of conventional tracking loops.

\section{SYSTEM MODEL}

\section{II-A. GNSS model}

Considering the signals coming from $M_{s}$ satellites in a multipath channel, the received signal is

$$
\begin{aligned}
r(t)= & \sum_{i=0}^{M_{s}-1} \sum_{m=0}^{M(i)-1} \alpha_{m, i}(t) \Re\left\{e^{j\left(2 \pi f_{c}\left(t-\tau_{m, i}(t)\right)+\phi_{m, i}\right)}\right. \\
& \left.\times s_{T, i}\left(t-\tau_{m, i}(t)\right)\right\}+w(t)
\end{aligned}
$$

where $\Re\{\cdot\}$ denotes the real part of the complex-valued quantity in the brackets, $M(i)$ is the number of propagation paths from the $i^{\text {th }}$ satellite to the receiver, $f_{c}$ is the 
carrier frequency, $\left\{\alpha_{m, i}(t), \tau_{m, i}(t), \phi_{m, i}\right\}$ are the amplitude, delay and carrier phase of the $i^{t h}$ satellite in the $m^{t h}$ path, $w(t)$ is additive white Gaussian noise, and $s_{T, i}(t)$ is the Direct-Sequence Spread-Spectrum (DS-SS) baseband signal transmitted by the $i^{\text {th }}$ satellite. In order to compute the position, the receiver should estimate the pseudoranges (directly related to time delay of the line-of-sight (LOS) paths, $\tau_{0, i}(t)$ ) and pseudorange rate (related to the evolution of the carrier phase). Considering the orthogonality between spreading codes, and the usual parallel architecture of a GNSS receiver, we will simplify the signal model to

$$
r(t)=\alpha_{0}(t) \Re\left\{e^{j\left(2 \pi f_{c}\left(t-\tau_{0}(t)\right)+\phi_{0}\right)} s_{T}\left(t-\tau_{0}(t)\right)\right\}+n(t)
$$

where the subindex 0 denotes the direct path, and $n(t)$ gathers all the unwanted signals (multiuser interference, multipath and termal noise) in a term which is considered Gaussian but not white, thus admitting a degree of correlation of the unwanted terms with the desired signal.

When this signal impinges the antenna, it passes through a Low Noise Amplifier (LNA) and a downconversion stage to an intermediate frequency. This can be modeled as

$$
y(t)=\Re\left\{\left(r(t) * h_{L N A}(t)\right) e^{-j\left(2 \pi f_{L O} t+\phi_{L O}\right)}\right\}
$$

where $*$ stands for the convolution operator, $h_{L N A}(t)$ is the impulse response for the RF front-end filter, $f_{L O}$ is the frequency of the local oscillator and $\phi_{L O}$ is its carrier phase. Defining $f_{I F}=f_{c}-f_{L O}, \psi_{0}=\phi_{0}-\phi_{L O}, s_{R}(t)$ as the filtered version of $s_{T}(t), a_{0}(t)$ as the signal amplitude, and $\eta(t)$ as the filtered and down-converted noise term $n(t)$, we can write

$y(t)=a_{0}(t) \Re\left\{s_{R}\left(t-\tau_{0}(t)\right) e^{j\left(2 \pi f_{I F} t-2 \pi f_{c} \tau_{0}(t)+\psi_{0}\right)}\right\}+\eta(t)$

Sampling this signal at $t_{k}=k T_{s}$, we obtain the discrete version of the received signal

$$
y_{k}=a_{0, k} \Re\left\{s_{R}\left(t_{k}-\tau_{0, k}\right) e^{j\left(2 \pi f_{I F} t_{k}-2 \pi f_{c} \tau_{0, k}+\psi_{0}\right)}\right\}+\eta_{k}
$$

where the subindex $k$ refers to $t_{k}$ instants.

Defining $\mathbf{p}^{(s)}$ and $\mathbf{p}_{(u)}$ as the position vector of the satellite and the user receiver, respectively, and neglecting atmospheric effects, the delay can be written as

$$
\tau_{0}(t)=\frac{1}{c}\left\|\mathbf{p}^{(s)}\left(t_{T_{x}}\right)-\mathbf{p}_{(u)}(t)\right\|=t-t_{T_{x}}
$$

being $c$ the speed of light, $t_{T_{x}}$ is the transmission instant, and $\|\cdot\|$ stands for the Euclidean norm. Approximating (6) by its second-order Taylor expansion around an arbitrary point $t_{0}$ we get

$$
\begin{aligned}
\| \mathbf{p}^{(s)}\left(t_{T_{x}}\right) & -\mathbf{p}_{(u)}(t)\|\approx\| \mathbf{p}^{(s)}\left(t_{0}-\tau_{0}\left(t_{0}\right)\right)-\mathbf{p}_{(u)}\left(t_{0}\right) \|+ \\
& +\frac{d}{d t}\left\|\mathbf{p}^{(s)}\left(t_{0}-\tau_{0}\left(t_{0}\right)\right)-\mathbf{p}_{(u)}\left(t_{0}\right)\right\|\left(t-t_{0}\right)+ \\
& +\frac{1}{2} \frac{d^{2}}{d t^{2}}\left\|\mathbf{p}^{(s)}\left(t_{0}-\tau_{0}\left(t_{0}\right)\right)-\mathbf{p}_{(u)}\left(t_{0}\right)\right\|\left(t-t_{0}\right)^{2}
\end{aligned}
$$

Substituting equations (7) and (6) into equation (5) yields

$$
y_{k}=a_{0, k} \Re\left\{s_{R}\left(t_{k}-\tau_{0, k}\right) e^{j\left(2 \pi \tilde{f}_{k} t_{k}+\tilde{\psi}_{k}\right)}\right\}+\eta_{k}
$$

where we have made use of the following definitions:

$$
\begin{aligned}
\tilde{f}_{k}= & f_{I F}-\frac{f_{c}}{c} v_{r} \\
\tilde{\psi}_{k}= & -\frac{2 \pi f_{c}}{c}\left(\left\|\mathbf{p}^{(s)}\left(t_{0}-\tau_{0}\left(t_{0}\right)\right)-\mathbf{p}_{(u)}\left(t_{0}\right)\right\|+\right. \\
& \left.-v_{r} t_{0}+a_{r}\left(t_{k}-t_{0}\right)^{2}\right)+\psi_{0} \\
v_{r}= & \frac{d}{d t}\left\|\mathbf{p}^{(s)}\left(t-\tau_{0}(t)\right)-\mathbf{p}_{(u)}(t)\right\|_{t=t_{0}} \\
a_{r}= & \frac{1}{2} \frac{d^{2}}{d t^{2}}\left\|\mathbf{p}^{(s)}\left(t-\tau_{0}(t)\right)-\mathbf{p}_{(u)}(t)\right\|_{t=t_{0}}
\end{aligned}
$$

Terms $v_{r}$ and $a_{r}$ are usually referred to as radial velocity and radial acceleration, respectively, while the term $f_{d}=\frac{f_{c}}{c} v_{r}$ is commonly known as the Doppler shift.

\section{II-B. INS model}

Inertial measurement units (IMUs) typically contain three orthogonal rate-gyroscopes and three orthogonal accelerometers, measuring angular velocity and linear acceleration respectively. Let us assume a strapdown INS system in with the inertial sensors are mounted rigidly onto the receiver device, and therefore outputs quantities measured in a body frame. A typical strapdown navigation algorithm works as follows: the rate-gyroscope measurements $\boldsymbol{\omega}_{b}(t)=$ $\left[\omega_{b_{x}}(t) \omega_{b_{y}}(t) \omega_{b_{z}}(t)\right]^{T}$ (that is, the angular velocity) are integrated to obtain the orientation of the tracked device. Rate-gyroscopes are known to suffer from bias, that is, the average output (usually given in ${ }^{o} / h$ ) from the gyroscope when it is not undergoing any rotation is not zero. A constant bias error, when integrated, causes an angular error which grows linearly with time. This effect can be modeled by a bias term, in the form

$$
\check{\boldsymbol{\omega}}_{b}(t)=\boldsymbol{\omega}_{b}(t)-\delta \boldsymbol{\omega}_{b}(t),
$$

where $\delta \boldsymbol{\omega}_{b}(t)=\left[\delta \omega_{b_{x}}(t) \delta \omega_{b_{y}}(t) \delta \omega_{b_{z}}(t)\right]^{T}$.

Since those measurements are given with respect to a body frame and we need them expressed in a global frame, a rotation matrix $\mathbf{C}_{b}^{g}$ such that $\check{\boldsymbol{\omega}}_{g}(t)=\mathbf{C}_{b}^{g} \breve{\boldsymbol{\omega}}_{b}(t)$ must be tracked through time. The rate of change of $\mathbf{C}_{b}^{g}$ at time $t$ is given by $\frac{d}{d t} \mathbf{C}_{b}^{g}(t)$. Using the small angle approximation, 
it comes out that the orientation algorithm must solve the differential equation

$$
\frac{d}{d t} \mathbf{C}_{b}^{g}(t)=\mathbf{C}_{b}^{g}(t) \boldsymbol{\Omega}(t)
$$

where $\boldsymbol{\Omega}(t)$ is the skew-symmetric form of the angular vector $\check{\boldsymbol{\omega}}_{b}(t)$ :

$$
\boldsymbol{\Omega}(t)=\left[\begin{array}{ccc}
0 & -\check{\omega}_{b_{z}}(t) & \check{\omega}_{b_{y}}(t) \\
\check{\omega}_{b_{z}}(t) & 0 & -\check{\omega}_{b_{x}}(t) \\
-\check{\omega}_{b_{y}}(t) & \check{\omega}_{b_{x}}(t) & 0
\end{array}\right]
$$

Taking into account that IMUs provide samples of the angular velocity at a constant rate of period $T_{i}$, equation (14) can be solved as

$$
\mathbf{C}_{b}^{g}\left(t+T_{i}\right)=\mathbf{C}_{b}^{g}(t) e^{\int_{t}^{t+T_{i}} \boldsymbol{\Omega}(t) d t}
$$

Solving the integral as $\int_{t}^{t+T_{i}} \boldsymbol{\Omega}(t) d t=\boldsymbol{\Omega}(t) T_{i}$ and performing a Taylor expansion of the exponential term yields

$$
\begin{aligned}
\mathbf{C}_{b}^{g}\left(t+T_{i}\right)= & \mathbf{C}_{b}^{g}(t)\left(\mathbf{I}-\frac{\sin \left(\left|\check{\boldsymbol{\omega}}_{b} T_{i}\right|\right)}{\left|\check{\boldsymbol{\omega}}_{b} T_{i}\right|} \boldsymbol{\Omega}(t) T_{i}+\right. \\
& \left.+\frac{1-\cos \left(\left|\check{\boldsymbol{\omega}}_{b} T_{i}\right|\right)}{\left|\check{\boldsymbol{\omega}}_{b} T_{i}\right|}\left(\boldsymbol{\Omega}(t) T_{i}\right)^{2}\right)
\end{aligned}
$$

To track the position of an INS the acceleration signal $\mathbf{a}_{b}(t)=\left[a_{b_{x}}(t) a_{b_{y}}(t) a_{b_{z}}(t)\right]^{T}$ obtained from the accelerometers is projected into the global frame of reference. Again, accelerometers are also biased. A constant bias error, when double integrated, causes an error in position which grows quadratically with time. This is modeled with a bias term

$$
\check{\mathbf{a}}_{b}(t)=\mathbf{a}_{b}(t)-\delta \mathbf{a}_{b}(t),
$$

where $\delta \mathbf{a}_{b}(t)=\left[\delta a_{b_{x}}(t) \delta a_{b_{y}}(t) \delta a_{b_{z}}(t)\right]^{T}$. Thus, the acceleration in a global frame can we written as $\check{\mathbf{a}}_{g}(t)=$ $\mathbf{C}_{b}^{g}(t) \check{\mathbf{a}}_{b}(t)$. Acceleration due to gravity is then subtracted and the remaining acceleration is integrated once to obtain velocity, and again to obtain displacement. In discrete form:

$$
\begin{aligned}
\mathbf{v}_{g, k} & =\mathbf{v}_{g, k-1}+T_{i}\left(\check{\mathbf{a}}_{g, k}-\mathbf{g}_{g}\right) \\
\mathbf{p}_{g, k} & =\mathbf{p}_{g, k-1}+T_{i} \mathbf{v}_{g, k}
\end{aligned}
$$

We also define i.i.d zero-mean Gaussian terms $\mathbf{w}_{\omega, k}$ and $\mathbf{w}_{a, k}$ modeling gyroscopes and accelerometers noise.

\section{II-C. GNSS/INS coupling}

We propose here a new GNSS/INS coupling approach, which consists on the derivation of the evolution of the synchronization parameters from the IMU measures. Taking into account the geometry of the satellite-user link, we can write that

$$
\begin{aligned}
\tau_{k}= & \tau_{k-1}-\frac{1}{c}\left(\mathbf{p}_{g, k}-\mathbf{p}_{g, k-1}\right)^{T} \mathbf{d}_{k-1}+ \\
& +\frac{1}{c}\left(\mathbf{p}^{(s)}\left(t_{k}-\tau_{k}\right)-\mathbf{p}^{(s)}\left(t_{k-1}-\tau_{k-1}\right)\right)^{T} \mathbf{d}_{k-1}+w_{\tau, k} \\
= & \tau_{k-1}+\beta_{k}^{(\tau)}+w_{\tau, k}
\end{aligned}
$$

where $\mathbf{d}_{k}$ is the unitary vector pointing from the user receiver to the satellite:

$$
\mathbf{d}_{k}=\frac{\mathbf{p}^{(s)}\left(t_{k}-\tau_{k}\right)-\mathbf{p}_{g, k}}{\left\|\mathbf{p}^{(s)}\left(t_{k}-\tau_{k}\right)-\mathbf{p}_{g, k}\right\|}
$$

The term $\mathbf{p}^{(s)}\left(t_{k}-\tau_{k}\right)$ represents the position from where the satellite emitted the signal received at $t_{k}$, an information that can be inferred from the navigation message. Since $\tau_{k}$ is what we are looking for, we make the approximation $\mathbf{p}^{(s)}\left(t_{k}-\tau_{k}\right) \approx \mathbf{p}^{(s)}\left(t_{k}-\tau_{k-1}\right)$. Proceeding in the same way, from (9) we can derive

$$
\begin{aligned}
\tilde{f}_{k}= & \tilde{f}_{k-1}+\frac{f_{c}}{c}\left(\left(\mathbf{v}^{(s)}\left(t_{k-1}-\tau_{k-1}\right)-\mathbf{v}_{g, k-1}\right)^{T} \mathbf{d}_{k-1}+\right. \\
& \left.-\left(\mathbf{v}^{(s)}\left(t_{k}-\tau_{k}\right)-\mathbf{v}_{g, k}\right)^{T} \mathbf{d}_{k}\right)+w_{f, k} \\
= & \tilde{f}_{k-1}+\beta_{k}^{(f)}+w_{f, k}
\end{aligned}
$$

where $\mathbf{v}_{g, k}$ is the velocity given by the IMU, and $\mathbf{v}^{(s)}(t)$ is the velocity of the satellite that can be extracted from the navigation message. Again, we will accept $\mathbf{v}^{(s)}\left(t_{k}-\tau_{k}\right) \approx$ $\mathbf{v}^{(s)}\left(t_{k}-\tau_{k-1}\right)$. The phase evolution can be predicted from (10) as

$$
\begin{aligned}
\tilde{\psi}_{k}= & \tilde{\psi}_{k-1}-\frac{2 \pi f_{c}}{c}\left(\left\|\mathbf{p}^{(s)}\left(t_{k}-\tau_{k}\right)-\mathbf{p}_{g, t_{k}}\right\|+\right. \\
& -\left\|\mathbf{p}^{(s)}\left(t_{k-1}-\tau_{k-1}\right)-\mathbf{p}_{g, t_{k-1}}\right\|+ \\
& +\left(\mathbf{v}^{(s)}\left(t_{k-1}-\tau_{k-1}\right)-\mathbf{v}_{g, k-1}\right)^{T} \mathbf{d}_{k-1} t_{0}+ \\
& -\left(\mathbf{v}^{(s)}\left(t_{k}-\tau_{k}\right)-\mathbf{v}_{g, k}\right)^{T} \mathbf{d}_{k} t_{0}+ \\
& -\left(\mathbf{a}^{(s)}\left(t_{k-1}-\tau_{k-1}\right)-\mathbf{a}_{g, k-1}\right)^{T} \mathbf{d}_{k-1}\left(t_{k-1}-t_{0}\right)^{2}+ \\
& \left.+\left(\mathbf{a}^{(s)}\left(t_{k}-\tau_{k}\right)-\mathbf{a}_{g, k}\right)^{T} \mathbf{d}_{k}\left(t_{k}-t_{0}\right)^{2}\right)+w_{\psi, k} \\
= & \tilde{\psi}_{k-1}+\beta_{k}^{(\psi)}+w_{\psi, k}
\end{aligned}
$$

where $\mathbf{a}_{g, k}$ is the acceleration measured by the IMU, and $\mathbf{a}^{(s)}(t)$ is the acceleration of the satellite, also extracted from the navigation message. Terms $w_{\tau, k}, w_{f, k}$ and $w_{\psi, k}$ are white Gaussian i.i.d. noise sequences.

We take a state-space approach in order to use an optimal Bayesian filtering solution. In equation (8), we have defined the discrete measurement equation. As we want to consider the biased behavior of the IMU, we introduce the bias of the rate-gyroscopes, $\delta \boldsymbol{\omega}_{b, k}$, and accelerometers, $\delta \mathbf{a}_{b, k}$, into the state, using a random walk evolution. We define the vector state

$$
\mathbf{x}_{k}=\left[\tau_{k}, \tilde{f}_{k}, \tilde{\psi}_{k}, \delta \boldsymbol{\omega}_{b, k}^{T}, \delta \mathbf{a}_{b, k}^{T}\right]^{T},
$$

and its evolution as

$$
\mathbf{x}_{k}=\boldsymbol{\Phi}_{k} \mathbf{x}_{\mathbf{k}-\mathbf{1}}+\mathbf{u}_{k}+\mathbf{w}_{k}
$$


where $\boldsymbol{\Phi}_{k}=\mathbf{I}_{9 \times 9}$ is the identity matrix, the input vector is $\mathbf{u}_{k}=\left[\beta_{k}^{(\tau)}, \beta_{k}^{(f)}, \beta_{k}^{(\psi)}, \mathbf{0}_{1 \times 6}\right]^{T}$, and the noise vector is $\mathbf{w}_{k}=\left[w_{\tau, k}, w_{f, k}, w_{\psi, k}, \mathbf{w}_{\omega, k}^{T}, \mathbf{w}_{a, k}^{T}\right]^{T}$.

\section{SIGMA-POINT NONLINEAR FILTERING}

When considering a nonlinear filtering problem, we can consider different suboptimal solutions. A common solution is the well-known EKF, a solution that is useful for mild nonlinearities but fails otherwise. Alternative solutions have been proposed in the last decade, among them, the family of Sequential Monte Carlo (SMC) methods [9] and the family of Sigma-Point Filters (SPFs) [10].

SMC methods provide a framework to deal with nonlinear, non-Gaussian problems using a stochastic sampling approach to numerically approximate the integrals of the optimal solution. This broad suitability comes at the expense of a high computational load, that makes this solution difficult to embed in digital light processors or real-time applications.

SPFs use a deterministic sampling to approximate the integrals involved in the Bayesian filter solution. The key point is to assume that the measurement and process noise are independent random Gaussian processes. This leads to Gaussian state transition and measurement likelihood densities, which in turn reverts to a Gaussian posterior density [11]. Recently, a new SPF method, and its squareroot version, has been derived: the Square-root Cubature Kalman Filter (SCKF) [8]. The SCKF uses cubature rules to approximate the optimal solution, and propagates the square root of the covariance matrix instead of the covariance itself, thus preserving its symmetry and positive-definiteness, and avoiding its inversion in the update step. The SCKF is numerically stable, accurate, and easily extendable to high dimensional problems, mitigating the curse of dimensionality and divergence effects.

We sketch in Algorithm 1 the square-root, derivativefree scheme. In steps 8 and $17, \mathbf{S}=\operatorname{Tria}(\mathbf{A})$ denotes a general triangularization algorithm (for instance, the QR decomposition), where $\mathbf{A} \in \mathbb{R}^{p \times q}, p<q$, and $\mathbf{S}$ is a lower triangular matrix. For computational reasons, we prefer to keep the square root as a triangular matrix of the dimension $p \times p$. This can be achieved by the thin QR decomposition [12, $\S 5.2$, Theorem 5.2.2], which has a computational complexity of $\mathcal{O}\left(q p^{2}\right)$ flops. Algorithm 2 provides the way to generate the deterministic samples, using the third-degree spherical-radial rule, to approximate a standard Gaussian weighted integral.

\section{COMPUTER SIMULATIONS}

The proposed integration solution was tested by computer simulation. We used a realistic GPS signal generator which considered aspects such as constellation geometry, receiver/satellites relative motion and time-varying $\overline{\text { Algorithm } 1 \text { Square-root, derivative-free nonlinear Kalman }}$ Filter

Require: $\mathbf{y}_{1: K}, \mathbf{u}_{0: K}, \hat{\mathbf{x}}_{0}, \boldsymbol{\Sigma}_{x, 0}=\mathbf{S}_{x, 0 \mid 0} \mathbf{S}_{x, 0 \mid 0}^{T}, \boldsymbol{\Sigma}_{w, 0}, \boldsymbol{\Sigma}_{\eta, 0}$.

\section{Initialization:}

1: Define sigma-points and weights $\left\{\boldsymbol{\xi}_{i}, \omega_{i}\right\}_{i=1, \ldots, L}$ by using Algorithm 2.

2: Set $\mathbf{W}=\operatorname{diag}\left(\sqrt{\omega_{i}}\right)$

\section{Tracking:}

3: for $k=1$ to $\infty$ do

4: Time update:

5: Evaluate the sigma points:

$\mathbf{x}_{i, k-1 \mid k-1}=\mathbf{S}_{x, k-1 \mid k-1} \boldsymbol{\xi}_{i}+\hat{\mathbf{x}}_{k-1 \mid k-1}, i=1, \ldots, L$.

6: Evaluate the propagated sigma points using (26):

$$
\tilde{\mathbf{x}}_{i, k \mid k-1}=\mathbf{f}\left(\mathbf{x}_{i, k-1 \mid k-1}, \mathbf{u}_{k-1}\right) \text {. }
$$

7: $\quad$ Estimate the predicted state:

$$
\hat{\mathbf{x}}_{k \mid k-1}=\sum_{i=1}^{L} \omega_{i} \tilde{\mathbf{x}}_{i, k \mid k-1} .
$$

8: Estimate the square-root factor of the predicted error covariance:

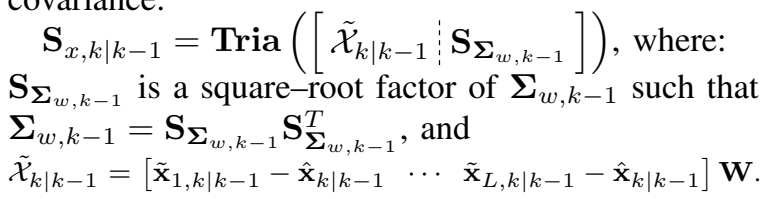

9: Measurement update:

10: Evaluate the sigma points:

$$
\mathbf{x}_{i, k \mid k-1}=\mathbf{S}_{x, k \mid k-1} \boldsymbol{\xi}_{i}+\hat{\mathbf{x}}_{k \mid k-1}, i=1, \ldots, L .
$$

11: Evaluate the propagated sigma points using (8):

$$
\tilde{\mathbf{y}}_{i, k \mid k-1}=\mathbf{h}\left(\mathbf{x}_{i, k \mid k-1}, \mathbf{u}_{k}\right) \text {. }
$$

12: Estimate the predicted measurement:

$$
\hat{\mathbf{y}}_{k \mid k-1}=\sum_{i=1}^{L} \omega_{i} \tilde{\mathbf{y}}_{i, k \mid k-1} .
$$

13: Estimate the square-root of the innovation covariance matrix:

$$
\mathbf{S}_{y, k \mid k-1}=\operatorname{Tria}\left(\left[\mathcal{Y}_{k \mid k-1} \mathbf{S}_{\boldsymbol{\Sigma}_{\eta, k}}\right]\right) \text {, where: }
$$

$\mathbf{S}_{\boldsymbol{\Sigma}_{\eta, k}}$ denotes a square-root factor of $\boldsymbol{\Sigma}_{\eta, k}$ such that $\boldsymbol{\Sigma}_{\eta, k}=\mathbf{S}_{\boldsymbol{\Sigma}_{\eta, k}} \mathbf{S}_{\boldsymbol{\Sigma}_{n, k}}^{T}$, and

$\mathcal{Y}_{k \mid k-1}=\left[\begin{array}{lll}\tilde{\mathbf{y}}_{1, k \mid k-1}-\hat{\mathbf{y}}_{k \mid k-1} & \cdots & \tilde{\mathbf{y}}_{L, k \mid k-1}-\hat{\mathbf{y}}_{k \mid k-1}\end{array}\right] \mathbf{W}$.

14: Estimate the cross-covariance matrix

$$
\boldsymbol{\Sigma}_{x y, k \mid k-1}=\mathcal{X}_{k \mid k-1} \mathcal{Y}_{k \mid k-1}^{T} \text {, where: }
$$

$\mathcal{X}_{k \mid k-1}=\left[\begin{array}{lll}\mathbf{x}_{1, k \mid k-1}-\hat{\mathbf{x}}_{k \mid k-1} & \cdots & \mathbf{x}_{L, k \mid k-1}-\hat{\mathbf{x}}_{k \mid k-1}\end{array}\right] \mathbf{W}$.

15: Estimate the Kalman gain

$$
\mathbf{K}_{k}=\left(\boldsymbol{\Sigma}_{x y, k \mid k-1} / \mathbf{S}_{y, k \mid k-1}^{T}\right) / \mathbf{S}_{y, k \mid k-1} \text {. }
$$

16: Estimate the updated state

$$
\hat{\mathbf{x}}_{k \mid k}=\hat{\mathbf{x}}_{k \mid k-1}+\mathbf{K}_{k}\left(\mathbf{y}_{k}-\hat{\mathbf{y}}_{k \mid k-1}\right) \text {. }
$$

17: Estimate the square-root factor of the corresponding error covariance:

18: end for

$$
\mathbf{S}_{x, k \mid k}=\operatorname{Tria}\left(\left[\mathcal{X}_{k \mid k-1}-\mathbf{K}_{k} \mathcal{Y}_{k \mid k-1} \mathbf{K}_{k} \mathbf{S}_{\boldsymbol{\Sigma}_{\eta, k}}\right]\right) .
$$

delay/Doppler signal parameterizations. In particular, we tested the tracking of the synchronization parameters of one satellite by a static receiver (considering the biases of a 
Algorithm 2 Generation of Sigma-Points and weights for third-degree spherical-radial cubature rule

1: Set $M=\operatorname{dim}(\mathbf{x})$ and $L=2 M$.

2: Set the cubature points $\boldsymbol{\xi}_{i}=\sqrt{M}\left[\mathbf{I}_{M \times M}-\mathbf{I}_{M \times M}\right]_{i}$, where $[\cdot]_{i=1, \ldots, L}$ indicates the $i$-th column.

3: Set the cubature weights $\omega_{i}=\frac{1}{2 M}, i=1, \ldots, L$.

typical MEMS-based IMU) over 1 second. The nominal Carrier-to-Noise density ratio was set to $C / N_{0}=46 \mathrm{~dB}$ $\mathrm{Hz}$, although we took into account that the instantaneous value of such parameter will depend on the elevation angle and on satellite's antenna radiation pattern. On the other hand, the considered GPS receiver had a pre-correlation filter of $2 \mathrm{MHz}$, a sampling frequency of $5.7143 \mathrm{MHz}$, an intermediate frequency of $4.308 \mathrm{MHz}$, and the tracked signal corresponded to that of the C/A code transmitted in the L1 link. With this setup, the receiver was able to track the synchronization parameters of the satellite, with an initial ambiguity given by the acquisition process, i.e., a standard deviation of half the sampling period. Figure 1 shows the Root Mean Squared Error (RMSE) results obtained with the SCKF in estimating the pseudorange. In Figure 1 we also plotted the Cramer-Rao Bound (CRB), which is the minimum variance that any unbiased estimator can achieve. The CRB in its standard formulation is valid for ML-like estimators, that is to say when no use of prior information is done. However, this is not our case since we are considering prior data, which is expressed in the state-evolution models proposed in Section II-C. Therefore, we must resort to the Bayesian CRB as a valid benchmark for our algorithm. From the figure it follows that the obtained pseudorange variances are in accordance to the theoretical bound. In addition, we can see the enhancement given by the use of prior information.

\section{CONCLUSIONS}

This paper presented a new approach to GNSS/INS integration. The main objective was to establish the pertinent mathematical relationships that allow the ultra-tight coupling of both systems, casting the problem into the Bayesian framework and proposing a solution based on Gaussian nonlinear filtering. Detailed computer simulations confirmed the validity of the approach.

\section{REFERENCES}

[1] Y. Yi amd D. A. Grejner-Brzezinska, "Tightly-coupled GPS/INS integration using unscented Kalman filter and particle filter," in Proc. ION GNSS, Fort Worth, TX, Sept. 2006, pp. 2182-2191.

[2] J. Rezaie, B. Moshiri, B. N. Araabi, and A. Asadian, "GPS/INS integration using nonlinear blending filters," in Proc. SICE'07, Kagawa, Japan, Sept. 2007, pp. 1674-1680.

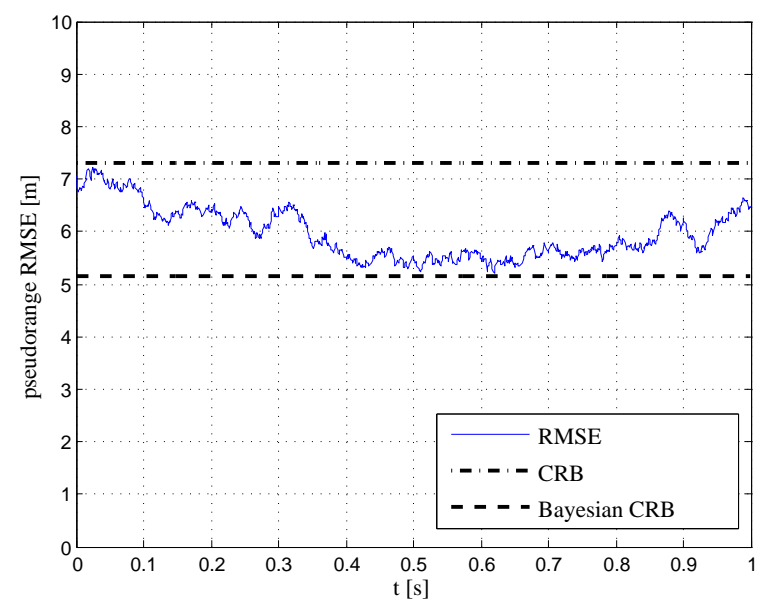

Fig. 1. RMSE of pseudorange estimates, $\mathrm{CRB}$ and Bayesian CRB over time.

[3] Y. Li, C. Rizos, J. Wang, P. Mumford, and W. Ding, "Sigma-point Kalman filtering for tightly coupled GPS/INS integration," Navigation, vol. 55, no. 3, pp. 167-178, Fall 2008.

[4] D. Bernal, P. Closas, E. Calvo, and J. A. FernándezRubio, "Tight GNSS/INS integration as a constrained least-squares problem," in Proc. EUSIPCO, Glasgow, Scotland, Aug. 2009, pp. 85-89.

[5] M.G. Petovello and G. Lachapelle, "Comparison of vector-based software receiver implementations with application to ultra-tight GPS/INS integration," in Proc. ION GNSS, Fort Worth, TX, Sept. 2006, pp. 17901799.

[6] D. Bernal, P. Closas, and J. A. Fernández-Rubio, "Particle filtering algorithm for ultra-tight GNSS/INS integration," in Proc. ION GNSS, Savannah, GA, Sept. 2008, pp. 2137-2144.

[7] R. Babu and J. Wang, "Ultra-tight GPS/INS/PL integration: a system concept and performance analysis," GPS Solutions, vol. 13, no. 1, pp. 75-82, Jan. 2009.

[8] I. Arasaratnam and S. Haykin, "Cubature Kalman Filters," IEEE Trans. Automatic Control, vol. 54, no. 6, pp. 1254-1269, June 2009.

[9] A. Doucet, N. De Freitas, and N. Gordon, Eds., Sequential Monte Carlo methods in practice, Springer, 2001.

[10] R. Van der Merwe, Sigma-point Kalman filters for probabilistic inference in dynamic state-space models, Ph.D. thesis, Oregon Health and Science University, Portland, OR, April 2004.

[11] K. Ito and K. Xiong, "Gaussian filters for nonlinear filtering problems," Trans. Automatic Control, vol. 45, no. 5, pp. 910-927, May 2000.

[12] G. H. Golub and C. F. van Loan, Matrix Computations, The John Hopkins University Press, 3rd edition, 1996. 